\title{
Shellhouse - [living portable]
}

\author{
Shellhouse - [living portable]
}

\section{$<$ Resumen>}

Las evoluciones sociales y económicas lideradas por Internet y las redes inalámbricas, cambiaron los conceptos espaciales y temporales que existían en el mundo para siempre. Nuestra sociedad consume estos avances que excluyen a grupos minoritarios, generando brechas aún más extensas entre primer y tercer mundo, pero también dentro de grupos sociales específicos. Shellhouse - living portable son refugios plegables de cartón que proveen de una dirección portátil a personas indigentes.

$<$ Abstract $>$

Social and economic evolutions lead by Internet and cordless networks have changed the concepts of space and time forever. Our society consumes these technological advances that exclude minority groups creating even wider gaps, not only between the First World and the Third World but also between specific social groups. Shellhouse - living portable are foldaway cardboard refuges that provide a portable address for homeless people.

<PALABRAS CLAVE>

ACCESO / TECNOLOGÍA / CONSUMO / REDES / MOVILIDAD / INTERNET / PARTICIPACIÓN

-KEYWORDS >

ACCESS / TECHNOLOGY / CONSUMERISM / NETWORKS / MOBILITY / INTERNET / PART ICIPATION

\section{Introducción}

El proyecto Shellhouse - living portable se genera a partir de un pensamiento que combina 1s y Os: acceso y marginalidad, abundancia y precariedad, consumo y desecho. Utilizando estas posibilidades de operación, la fusión de arte y tecnología, Shellhouse - living portable plantea interrogantes sobre refugio, movilidad, dirección, locación, sustentabilidad y desarrollo de tecnología al alcance de sectores en la población que podrían ser integrados sin necesidad de cambiar sus hábitos de habitación, pero haciéndolos visibles. Fue generado en la ciudad de Nueva York el año 2007 y ha evolucionado según distintas colaboraciones alrededor del mundo.
En su primera versión, Shellhouse - living portable era un proyecto construido a base cartón recogido en las calles como símbolo de desecho (por lo tanto de consumo) y dispuesto en las veredas de Nueva York en grandes cantidades, construyendo una gran plancha que era plegada bajo técnicas de origami y que recibía señales de radio a través de dispositivos puestos en cada refugio que emitían señales a una central, al que encendía un equipo MP3 con muestras sonoras de datos de los ocupantes de los refugios (nombre, edad, lugar de origen). En su segunda versión, el proyecto siguió teniendo el mismo diseñó para los refugios, pero el circuito en cada uno de ellos, emitía señales de radio a una central que generaba, inalámbricamente, cambios de color en una pantalla, según el ocupante del refugio

\footnotetext{
Artista egresada de la Facultad de Artes de la Universidad de Chile, con estudios de posgrado en Gestión Cultural en la Pontificia Universidad Católica de Chile y en la Facultad de Arte y Arquitectura de Columbia University. Máster del Programa de Telecomunicaciones Interactivas de la Universidad de Nueva York. Actualmente es académico en el Magíster la Facultad de Arquitectura de la Pontificia Universidad Católica, Profesor de Taller de cursos superiores de la Facultad de Artes de la Universidad de las Comunicaciones, UNIACC y dicta talleres de Physical Computing en la Facultad de Artes de la Universidad de Chile y otros espacios académicos.
} 
apretaba un botón de los dos posibles en el dispositivo.

Las instrucciones para construir tanto el refugio como el circuito, estuvieron siempre on-line para que usuarios comunes y corrientes, organizaciones u otros, pudieran construir su propia red o sumarse a la existente.

En futuras versiones Shellhouse - living portable contempla la comunicación P2P entre refugios y hacia el nodo central, sin alterar la simpleza del circuito, su tamaño, ni el material del refugio.

\section{Propiedad, ubicación y tecnología}

Estilos de vida contemporánea replantean la presente crisis de la metrópolis posmoderna. La reorganización de la sociedad ha dado prioridad entre arquitectos a necesidades inducidas, típicas de una sociedad postindustrial más que de necesidades elementales. La fragmentación de la sociedad y los cambios que han aparecido debido a la presencia de comunidades migratorias dinámicas, desafían estilos de vida monoculturales previos. Una sociedad ordenada y dividida en clases ha sido reemplazada por una sociedad compuesta de grupos, una realidad nueva y multifacética.

El racionalismo de las primeras décadas del siglo xx desarrolló la tendencia de equilibrar la función de vivir con funciones mecánicas. Justo cuando la industria automovilística producía autos masivamente, también una nueva industria de construcción sería equipada para producir casas en serie. La «típica casa reflejaba la clasificación de la sociedad y era definida por la economía. El hecho de que ta clasificación no se aplica más en la sociedad del presente, es claramente visible en la falta de respuesta de las áreas de un público poco deseoso de identificarse con estilos de vida ofrecidos por el mercado.

La creciente conciencia de un mundo en el que se construye considerando los limitados recursos naturales, los requerimientos de una compatibilidad ambiental entre construcción y naturaleza, y la necesidad de inventar tipos de espacio que son más flexibles y efímeros, más reducidos que cohabitan con estilos de vida menos reposados y más móviles, generan nuevas respuestas y soluciones para estilos de vida temporales, refugios de emergencia, casas «hágalo usted mismo», hasta aquéllas para usuarios especiales (estudiantes, casas para madres solteras, para poblaciones

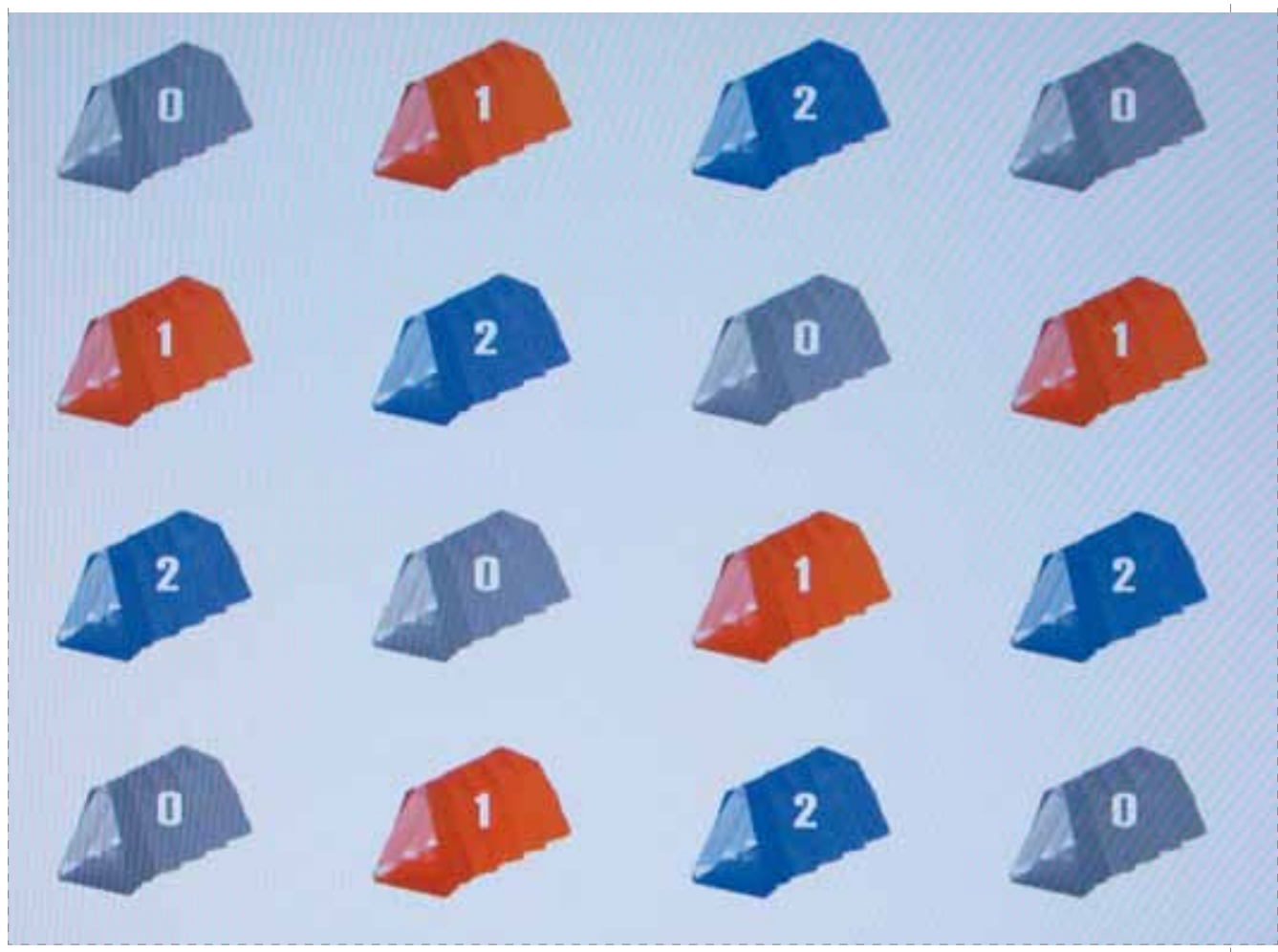

flotantes, trabajadores estacionarios, etc.). Aún como proyectos experimentales, tendencias de escuelas y también tomado como tema en el trabajo de artistas contemporáneos, conceptos como propiedad, espacio y nomadismo, al fin sitúan a la población dentro de la utopía que Cage y Fuller ya proponían en los '60. Un trabajo interdisciplinario en pos de una mente universal, positivo, en donde el caos y la arbitrariedad, en conjunción con el uso de tecnologías, generarían el nuevo arte y los cambios reales en la sociedad.

\section{La naturaleza del espacio social, el virtual y la propiedad}

El espacio virtual, con juegos como Second $L$ ife, en donde se entremezclan realidad «real» con «virtual», se transforman en una segunda oportunidad de vivir y habitar espacios virtuales: con tu tarjeta de crédito compras una casa que puedes habitar y decorar cada vez que ingresas al juego, invitas gente a ella, tienes citas con otras personas y la ropa que vistes se va ensuciando y desgastando... Los enormes cambios en los comportamientos humanos sociales que generan instancias como éstas alrededor del mundo, o las mismas redes sociales, en donde grupos de personas, unidas bajo un pensamiento similar, se unen en comunidades y existen en ese espacio virtual bajo una legalidad «real». En la era de la revolución de las telecomunicaciones, Napster Instructibles, Flickr, Facebook o Youtube son espacios donde el tiempo se considera en su única condición posible: el «ahora». La información dispuesta para todos de una manera instantánea y de difusión múltiple.

El Arte ha tomado estos conceptos e invadido terrenos que antes les eran propio a la Ciencia y Matemática, utilizando tecnologías avanzadas para plantearlas como medio, instalando nuevas interrogantes y métodos representativos sin tomar el lugar de la pintura, la escultura o la fotografía, convirtiéndose «en otro», una disciplina híbrida, que conjuga tiempo, espacio y materia desde diversos planos, o más bien, desde diversas herramientas tecnológicas como software y hardware desarrollados en su mayoría, para uso libre. Tal desarrollo ha revolucionado las técnicas utilizadas no sólo en Arte, también en Arquitectura, Diseño y otras áreas Humanistas y Científicas que han visto cuestionadas su técnicas educativas, debido a que la propagación del aprendizaje de ciertas herramientas que antes requerían de personas para poder ser adquiridas y que necesariamente, remitían a la propiedad (de autor, de tangibles, de conocimiento), un concepto que se va relativizando según se 

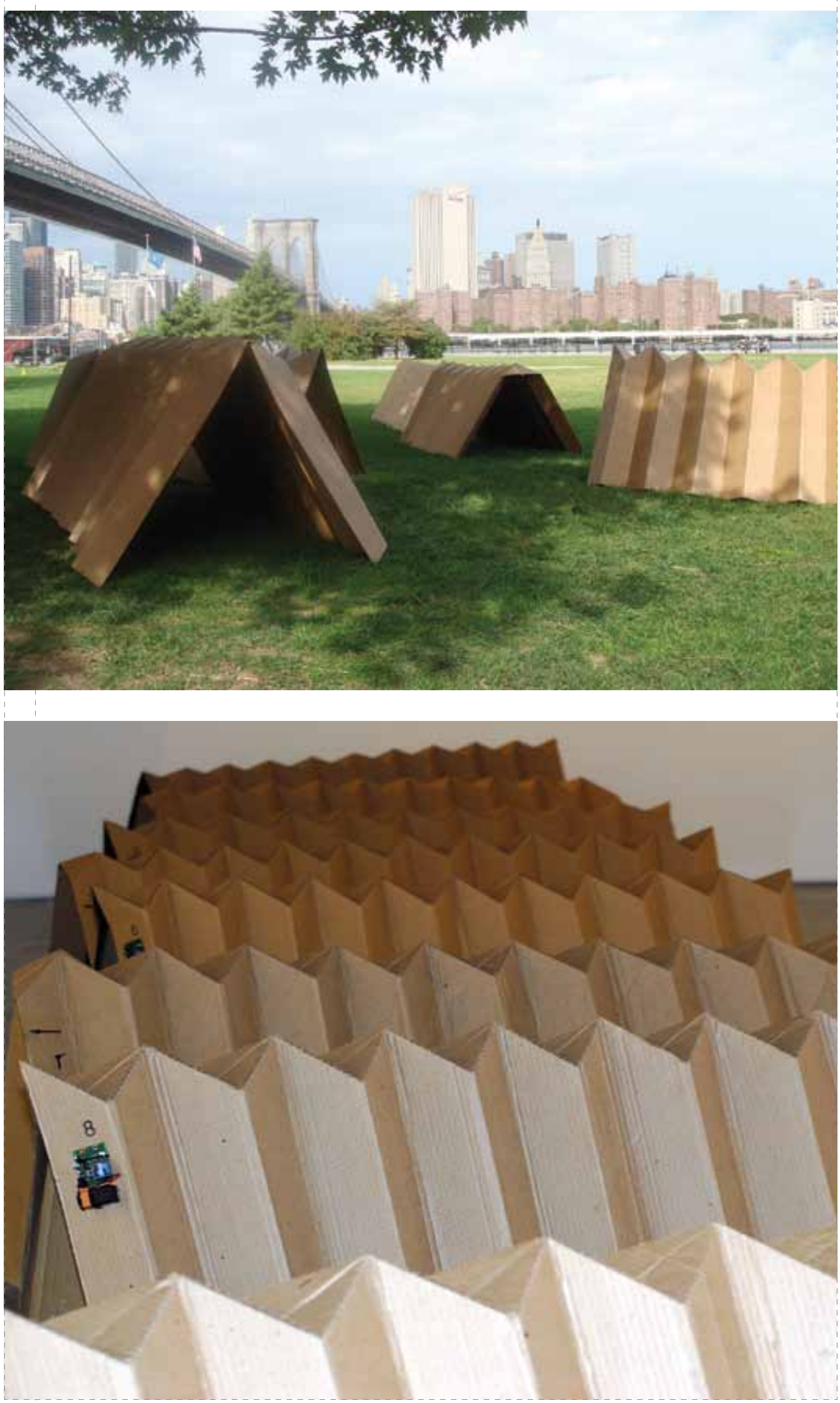

desarrollan acuerdos entre los usuarios de la tecnología.

Propiedad e identidad se van entrelazando: la propiedad ahora va constituyendo una relación y ya no sólo en un elemento objetivable. La noción de propiedad se une al concepto de uso, de consumo (no es lo que uno posee lo que contribuye, sino lo que uno consume. Esto explica el porqué el sector de mayor crecimiento de la economía de consumo es la información. Un intangible que no puede ser poseído, ni adeudado). Las leyes existen para proteger la propiedad, para dar confianza que la cosa que uno posee, permanecerá disponible en el futuro. Cada vez más, nuestra economía se basará en productos que no pueden ser adquiridos y se transformarán más en asunto conceptual como ownerships, tomando algunas leyes aparatosas reconocidas como obstáculos. Este descanso en la protección y la propiedad se aplica a cualquiera que posea algo, cualquier cosa, desde una gran fortuna hasta un oxidado automóvil. Pero cuando la amenaza de privación es removida, la propiedad no es ya necesaria y las leyes se hacen inservibles. Un buen ejemplo de esto es el carro de supermercado: nadie siente la necesidad de poseer uno porque hay muchos de ellos disponibles para el uso común.

\section{Ubicación: Seguridad global y sustentabilidad}

\section{Migración Global y Movilidad Humana}

Mientras que la migración es un fenómeno antiguo, la escala corriente y características particulares lo hace un tópico global emergente en el siglo xxı.Inmigración en los EE.UU. y en Europa Occidental son parte de un sistema global de población en movimiento. A través del mundo, unos 200 millones de personas están ahora viviendo fuera de su país de origen. Las migraciones, dadas por oportunidades económicas, tumultos políticos, reunificación familiar, guerra y crisis ambientales, usan transportación contemporánea y tecnologías de la comunicación para mantener lazos familiares y el movimiento de ida y venida, la «migración circular», provocando profundos efectos económicos, sociales y culturales en su país de origen, tránsito y destino. Mientras la globalización ha hecho posible que las barreras a movimientos internacionales sean cada vez más bajos en cuanto a los bienes y el capital, el movimiento de las personas es aún sujeto oficial para amarrar el control. 


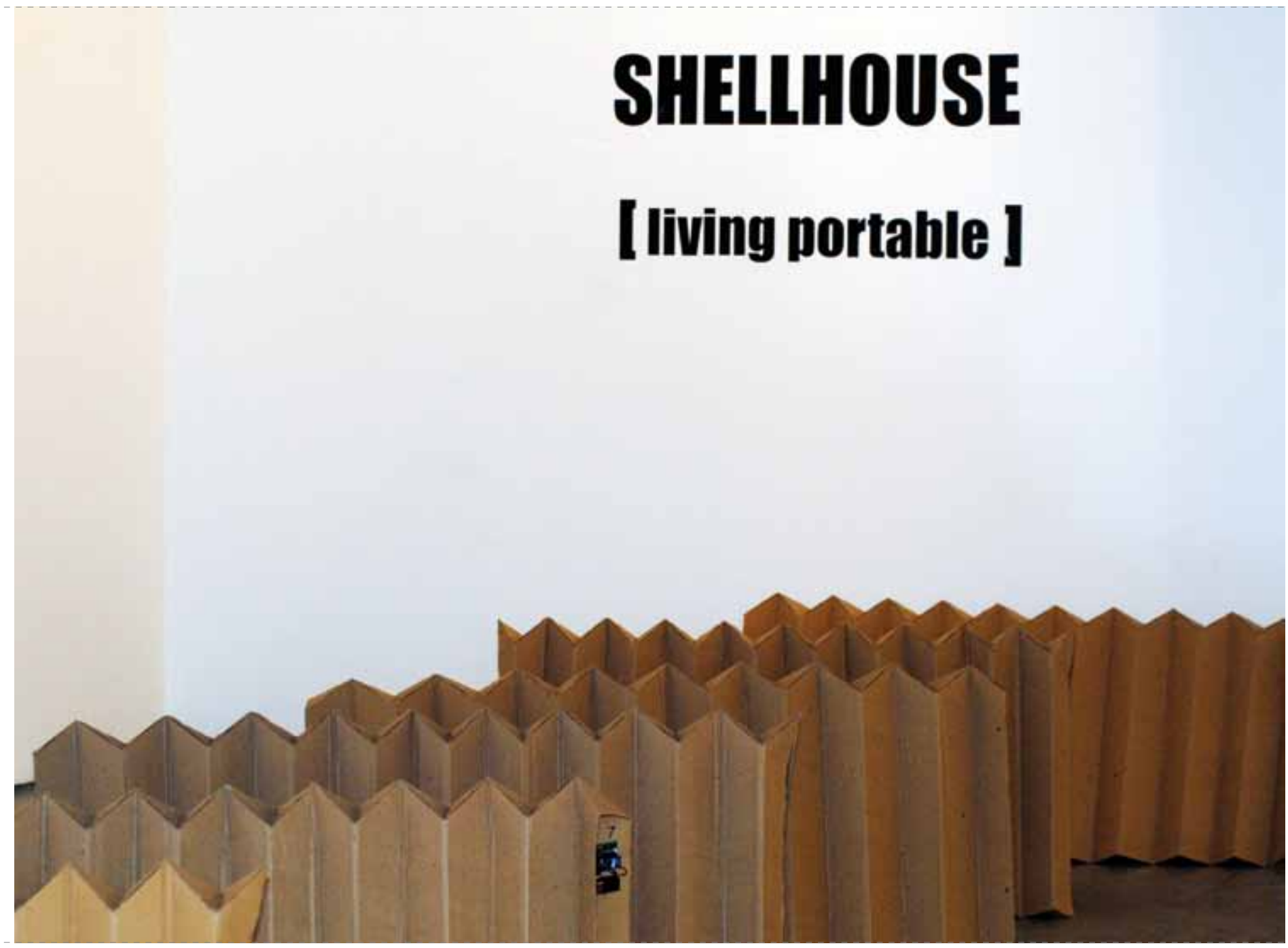

\section{Tecnología Ubicua}

Podemos desconectar el computador, calentar la comida en olla a fuego, dejar el celular en casa y en definitiva, prescindir de la tecnología que usamos a diario, pero no podríamos escapar de la omnipresente: cámaras de vigilancia en supermercados, bancos y algunas calles de la ciudad, uso de tarjetas de crédito, registro de datos y transportes almacenados como historiales personales. La taza del café y la pintura de los muros, prometen sensar y procesar información en el futuro.

En ciudades de alto desarrollo tecnológico como Tokio y Nueva York, podemos encontrar complejos sistemas de redes, uso de RFID (identificación por radio frecuencia), GPS (sistema de posición global) y mesh networking (mallas de redes) que, en diferentes escalas controlan flujos de movimiento, locación, identidad y disponibilidad. La conciencia que tenemos al interactuar en la vida a diario con objetos cotidianos como manillas para subir la escalera, altura de cada peldaño, ángulo de los respaldos de la sillas que utilizamos, mangos de cubiertos, situaciones de diseño variadas, que se desplazan también a comportamientos humanos, tonos de voz, gestos y comportamientos con otras personas, tenderán a desaparecer cuando la tecnología invisible se hagan presente. El diseño desaparecerá en el comportamiento. No es imposible pensar en que hablar por celular será sin duda, sólo hablar, el objeto celular desaparecerá y no precisamente porque se hará mas ergonométrico (esto se hace evidente en el Nokia Morph Concept) o delgado, sino porque se hará completamente invisible. Tales interacciones serán sentidas o vividas por nosotros como «correctas» $y$ «humanas» y el objeto se hará invisible.
Asuntos como el acceso no deberán entonces, ser puntos neurálgicos, porque situaciones de exclusión serán imposibles en un futuro positivo y optimista que considera a todos en usos y aplicaciones tecnológicas en resolver problemas de necesidad básica como alimento y vivienda.

\section{Bibliografía}

Virilio, P. «Open Sky», London, New York: Verso. 1997.

Greenfield, A. «Everyware: The dawning age of ubiquitous computing», USA: New Readers. 2006.

Bauman, Z. «Liquid Modernity», USA. Polity Press and Blackwell Publishers Ltd. 2000.

Sachs, J. «The End of Poverty», USA. The Penguin Press. 2005. 